\title{
The effectiveness of tensioning neural mobilization of brachial plexus in patients with chronic cervical radiculopathy: a randomized clinical trial
}

\author{
DOI: https://doi.org/10.5114/pq.2020.96419
}

\author{
Asmaa Omer Ibrahim', Nadia Abdelazeem Fayaz², Ahmed Hazim Abdelazeem ${ }^{3}$, Karima Abdelaty Hassan² \\ ${ }^{1}$ Ministry of Health and Population, Cairo, Egypt \\ ${ }^{2}$ Department of Physical Therapy for Musculoskeletal Disorders, Faculty of Physical Therapy, Cairo University, Egypt \\ ${ }^{3}$ Department of Orthopedic Surgery, Faculty of Medicine, Cairo University, Egypt
}

\section{Abstract}

Introduction. Tensioning neural mobilization (NM) is accomplished through increasing the distance between nerve bed ends via elongation. NM techniques have different biomechanical effects on the nervous system. Evidence for their use in treating certain upper-quarter conditions like cervicobrachial pain is limited. The study was to determine tensioning NM efficacy on unilateral chronic cervical radiculopathy regarding mechanosensitivity of the affected nerve roots and intensity of neck and arm pain.

Methods. Forty participants with chronic unilateral cervical radiculopathy were randomly assigned to group $\mathrm{A}(n=20)$, receiving traditional physical therapy (manual traction and infrared irradiation), and group $\mathrm{B}(n=20)$, receiving traditional physical therapy in addition to tensioning NM of brachial plexus. Mechanosensitivity of the affected brachial nerve roots and intensity of neck and arm pain were evaluated at baseline and after a 3-week program with the upper limb tension test-1 and visual analogue scale. The normal $(Z)$ test, paired and unpaired $t$-test, Wilcoxon signed-rank test, and Wilcoxon rank-sum test (Mann-Whitney) were used in data analysis.

Results. There were significant within-group differences in both groups regarding mechanosensitivity and pain intensity (mechanosensitivity: $p=0.001$ for group A, $p=0.001$ for group B; pain: $p<0.01$ for group $\mathrm{A}, p<0.01$ for group $\mathrm{B}$ ). There was no statistically significant between-group difference regarding mechanosensitivity $(p=0.07)$ or pain intensity $(p=0.838)$.

Conclusions. The addition of tensioning NM to traditional physical therapy had no significant benefits, although both groups showed decreased post-treatment mechanosensitivity and pain intensity.

Key words: chronic cervical radiculopathy, neural mobilization, mechanosensitivity

\section{Introduction}

Cervical radiculopathy $(\mathrm{CR})$ is a pain and/or sensorimotor deficit syndrome caused by compression of a cervical nerve root. The compression may result from disc herniation, spondylosis, instability, trauma, or tumours. The patient may complain of pain, numbness, and/or tingling in the upper extremity, which may develop to electrical-type pains or even weakness [1].

Symptoms of mechanically induced nervous system disorders can have either local or remote causes. The local causes include hypoxic, damaged, regenerating, or immature axons, connective tissue irritation (e.g. dura mater, epineurium, attachments), and mechanical interface irritation, while the remote causes are extra-segmental referral from the dura mater and nerve root sleeves, mechanical interface referral (e.g. disc, zygapophyseal joint), and referral from the autonomic nervous system. Intraneural pathology will affect the intrinsic elasticity of the nervous system and extra-neural pathology will affect the nerve/interface relationship [2].

Changes in the neurophysiology and mobility of the peripheral nerves usually cause neural dysfunction [3]. These changes can lead to mechanosensitivity, which is the sensitivity of a nerve to movement and palpation, resulting in pain during movement and/or sustained postures [4].

Tensioning neural mobilization (NM) is accomplished through increasing the distance between each end of the nerve bed through elongation [5]. NM techniques, including tensioning techniques, have different biomechanical effects on the nervous system $[6,7]$. There is limited evidence for the use of NM in the treatment of certain upper-quarter conditions [8]. Also, few studies focused on the effect of tensioning $\mathrm{NM}$ on mechanosensitivity in patients with $\mathrm{CR}$. The purpose of this study was to investigate if there were any benefits from adding tensioning NM to a traditional physical therapy program in the treatment of patients with chronic unilateral $\mathrm{CR}$ regarding mechanosensitivity and pain intensity.

\section{Subjects and methods}

\section{Patients}

Forty (male and female) patients with unilateral CR participated in the study and were randomly assigned to group $A$ $(n=20)$, who received traditional physical therapy in the form of manual traction and infrared irradiation, and group $\mathrm{B}(n=$ 20 ), who received traditional physical therapy in addition to tensioning NM of brachial plexus. All patients were informed about the study procedures and all patients were referred by physicians for physiotherapy. The study was conducted at the physiotherapy outpatient clinic in Al-Nozha hospital.

Patients were included if they were $20-40$ years of age, had a history of pain for more than 3 months, had radiating pain only in one upper limb, and met at least 3 of the Wainner

Correspondence address: Asmaa Omer Ibrahim, Physical Therapist at the Ministry of Health and Population, 5 Al-Eman St, New-Nozha 2, Cairo, Egypt, e-mail: asmaa84551@gmail.com 
et al. [9] criteria. Those who had a history of high level spinal cord injury and malignancy or any of the medical 'red flags' (e.g. tumour, fracture, rheumatoid arthritis, osteoporosis, prolonged steroid use), circulatory disturbances of upper extremity, traumatic injuries of upper limb and cervical spine, or dizziness were excluded from the study [10]. Also, patients were excluded if they had any contraindications to any of the applied treatment programs.

\section{Randomization}

After baseline evaluation, the patients were randomly assigned to 2 groups by using opaque, sealed envelopes, each containing the name of one of the groups (traditional physical therapy or NM). The envelopes were picked before the first treatment session by an investigator not participating in the study.

\section{Sample size calculation}

Sample size calculation was based on a previously reported difference of pain and disability in a study by Anwar et al. [11], 2-tailed alpha level of 0.05 , and $80 \%$ power. A sample size of 20 patients per group was determined.

\section{Assessment procedures}

The participants' demographic data, including name, age, height, and weight, were recorded. An investigator who was blind to the group assignment and was not involved in the intervention was responsible for the assessment before starting the treatment (baseline) and at 3 weeks after the intervention.

The upper limb tension test-1 (ULTT-1) was used to assess the mechanosensitivity of brachial nerve roots. ULTT-1 is evident to be reliable, valid, and responsive in evaluating the outcome in persons with CR [12].

The patient was supine without a pillow, and the hand of the untested side rested on the abdomen. The standardized sequence of upper limb movements was performed as follows: shoulder girdle depression, $90^{\circ}$ shoulder abduction, wrist and fingers extension, forearm supination, $90^{\circ}$ shoulder external rotation, and, finally, elbow extension [12].

Full range of each movement was reached unless the patient's symptoms reproduced. Any sensation such as stretch, tingling, or pain anywhere in the arm or neck was communicated with the patient. If any of these sensations were provoked, structural differentiation between neurogenic and nonneurogenic sources of pain was performed by the addition of sensitizing movements at a site distant to the pain: ipsi- and contralateral cervical lateral flexion, shoulder girdle elevation, wrist extension, or wrist flexion. More than one of these sensitizing movements may be used to obtain a clear patient's response. The unaffected arm response to the test was used as a reference for the affected arm. ULTT- 1 was considered positive if it reproduced the patient's symptoms at least partially and if the structural differentiation supported a neurogenic source [12].

Neck and arm pain were assessed by using a visual analogue scale. The subjects were instructed to mark any point on the continuum that expressed their pain intensity along the neck and the affected arm. The measurement of the length was then recorded as pain intensity [13].

\section{Treatment procedures}

Both groups received traditional physical therapy consisting of infrared radiation and manual traction. NM was additionally applied in group $\mathrm{B}$. The rehabilitation program was performed for all patients, 3 sessions per week, under the supervision of the principal investigator for 9 sessions.

\section{Infrared radiation}

The application parameters were as follows: model $2004 / 2 \mathrm{~N}$, power of $400 \mathrm{~W}$, voltage of $203 \mathrm{~V}$, and frequency of $50 / 60 \mathrm{~Hz}$. With the patient in a sitting position and their head supported comfortably over a pillow on the top of a table, the neck was exposed and the infrared was adjusted with the centre of the emission coil directly above and behind the spinous process of the $4^{\text {th }}$ cervical vertebra. The distance between the patient and the lamp was adjusted so that the patient reported mild comfortable warmth over the back of their neck. The irradiation time was 20 minutes [14].

\section{Manual traction of the cervical spine}

With the patient in a supine position, the therapist applied distraction force by placing the right hand on the patient's chin and left hand on the occiput; then, the distraction force was applied for 15 seconds for 3 sets of 10 repetitions, with 30 - and 60-second rest between repetitions and sets, respectively [15].

\section{Neural mobilization}

Group B received the same program as mentioned above, in addition to tensioning NM of brachial plexus $[16,17]$. The patient was supine, with their arm passively positioned in the neurodynamic testing position, $25^{\circ}$ of contralateral cervical lateral flexion, followed by passive scapular depression, $90^{\circ}$ combined shoulder abduction and external rotation, forearm supination, wrist extension, and, finally, finger extension (Figure 1). Movements were performed to full range or until a sense of resistance perceived by the investigator.

After being in the previous neurodynamic testing position, the patient's arm was taken into passive elbow extension for

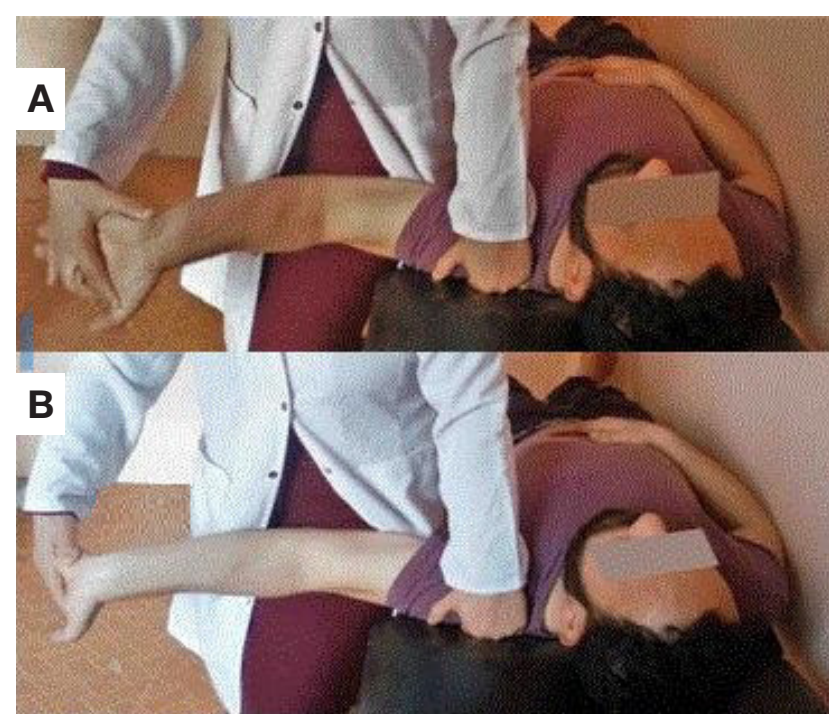

Figure 1. Tensioning neural mobilization of brachial plexus. A: the starting neurodynamic testing position B: taking patient's elbow into extension 
10 cycles of elbow extension and flexion for 6 seconds ( 3 seconds into extension and 3 seconds into flexion). The initial sense of resistance perceived by the investigator was used as a sign to alternate directions when moving from elbow flexion to extension. Finally, at the $10^{\text {th }}$ cycle, static hold was maintained while in elbow extension for 10 seconds $[16,17]$.

\section{Statistical analysis}

Data were analysed with the Shapiro-Wilk W test and by using the SPSS software, version 22.0 (SPSS Inc., Chicago, USA), in accordance with the statistical distribution. Descriptive statistics were obtained for variables (age, height, weight) as means and standard deviations, unpaired $t$-test was applied for within- and between-group data analysis of sample background (age, height, and weight), normal ( $Z$ ) test served for mechanosensitivity data analysis. The Mann-Whitney test and Wilcoxon signed-rank test were used for within- and between-group data analysis of pain intensity. The alpha level was set at 0.05 .

\section{Ethical approval}

The research related to human use has complied with all the relevant national regulations and institutional policies, has followed the tenets of the Declaration of Helsinki, and has been approved by the Research Ethical Committee of Faculty of Physical Therapy, Cairo University, Egypt (No.: P.T.REC/ 012/001656).

\section{Informed consent}

Informed consent has been obtained from all individuals included in this study.

\section{Results}

Overall, 76 patients were screened for inclusion criteria, 10 refused to participate in the study, 26 did not meet the inclusion criteria, and 40 patients were allocated into 2 groups (Figure 2).

There was no statistically significant difference $(p>0.05)$ in age, height, or weight for patients in the $A$ and $B$ groups, with the $p$ value equal $0.455,0.343$, and 0.752 for the respective parameters.

Regarding mechanosensitivity, there was no statistically significant difference between groups $A$ and $B(p=0.07)$, but for within-group data; there was a statistically significant difference between treatments (before and after treatment) for group A and group B, as shown in Table 1.

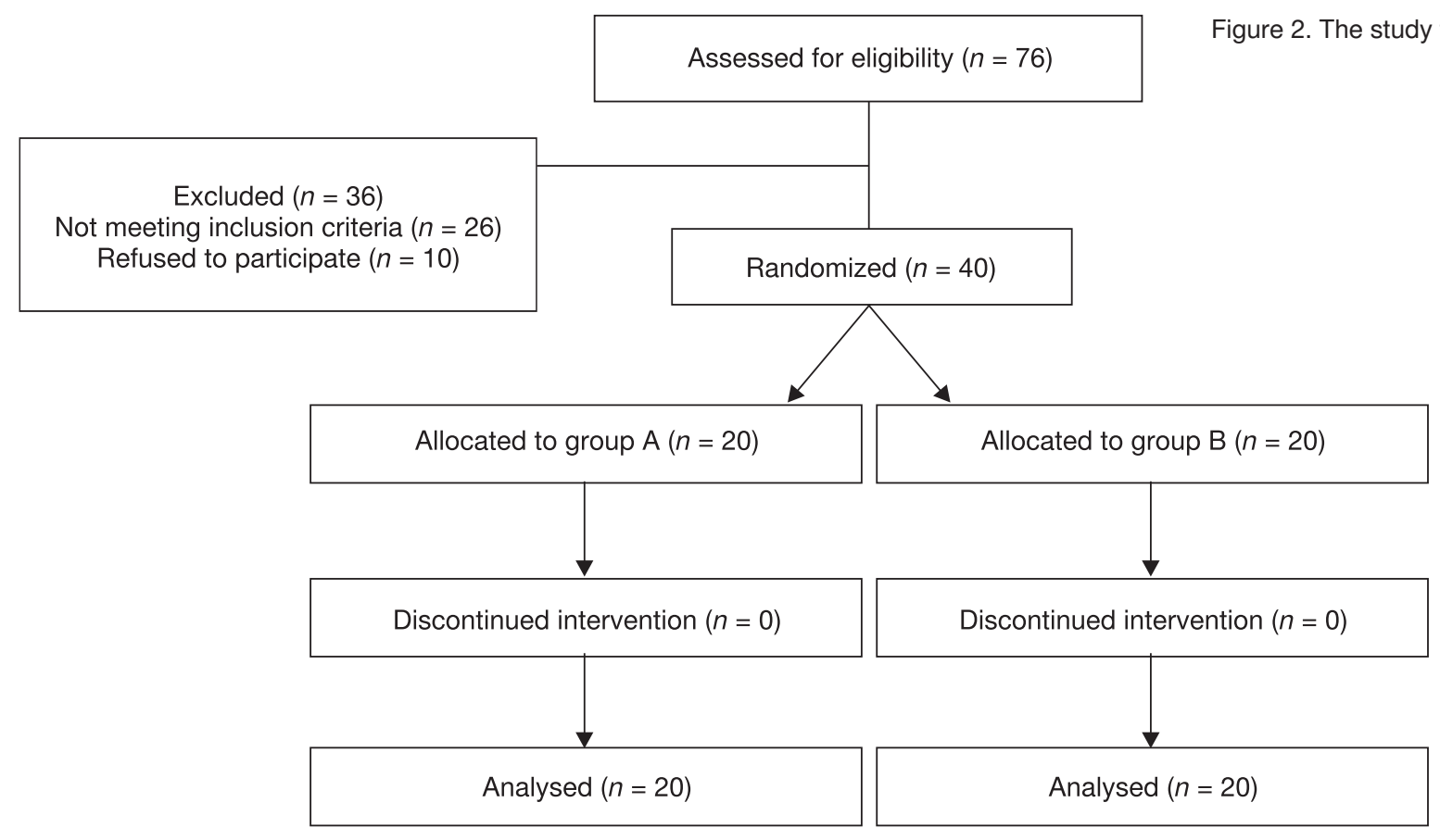

Table 1. Within- and between-group differences for mechanosensitivity

\begin{tabular}{|c|c|c|c|c|c|c|c|c|c|}
\hline & & & e tre & & & treat & & Witl & oups \\
\hline & & $n$ & $x$ & $\%$ & $n$ & $x$ & $\%$ & 7 & $p$ \\
\hline Group A & Positive & 20 & 18 & 90 & 20 & 9 & 45 & 3.464 & $0.001^{*}$ \\
\hline$(n=20)$ & Negative & 20 & 2 & 10 & 20 & 11 & 55 & 3.464 & $0.001^{*}$ \\
\hline Groun B & Positive & 20 & 16 & 80 & 20 & 2 & 10 & 5.534 & $0.001^{*}$ \\
\hline$(n=20)$ & Negative & 20 & 4 & 20 & 20 & 18 & 90 & 5.534 & $0.001^{*}$ \\
\hline Between & $Z$ & & 0.79 & & & 1.75 & & & \\
\hline groups & $p$ & & 0.12 & & & 0.07 & & & \\
\hline
\end{tabular}

ULTT-1 - upper limb tension test-1, $n$ - sample size, $x$ - number of patients

* significant at the level of $p<0.01$ 
Table 2. Within- and between-group differences for VAS using Mann-Whitney test and Wilcoxon signed-rank test $(n=20)$

\begin{tabular}{|c|c|c|c|c|c|c|c|c|}
\hline \multirow{2}{*}{\multicolumn{2}{|c|}{ VAS }} & \multicolumn{2}{|c|}{ Before treatment } & \multicolumn{2}{|c|}{ After treatment } & \multicolumn{3}{|c|}{ Within groups } \\
\hline & & Median & Range & Median & Range & $Z$ & $p$ & Indication \\
\hline \multicolumn{2}{|l|}{ Group A } & 7.50 & 7 & 3.75 & 7 & 4.36 & $0.001^{*}$ & $S$ \\
\hline \multicolumn{2}{|l|}{ Group B } & 7.50 & 6 & 3.75 & 7 & 4.60 & $0.001^{*}$ & $S$ \\
\hline \multirow{3}{*}{$\begin{array}{l}\text { Between } \\
\text { groups }\end{array}$} & $z$ & \multicolumn{2}{|c|}{0.150} & \multicolumn{2}{|c|}{0.204} & & & \\
\hline & $p$ & \multicolumn{2}{|c|}{0.881} & \multicolumn{2}{|c|}{0.838} & & & \\
\hline & Indication & \multicolumn{2}{|c|}{ NS } & \multicolumn{2}{|c|}{ NS } & & & \\
\hline
\end{tabular}

VAS - visual analogue scale, S - significant, NS - not significant

* significant at the level of $p<0.01$

With reference to pain intensity, there was no statistically significant difference between groups $A$ and $B(p=0.838)$, but for within-group data; there was a statistically significant difference between treatments (before and after treatment) for group A and group B, as shown in Table 2.

\section{Discussion}

The results of this study revealed that there was a decrease in mechanosensitivity of the affected brachial nerve roots and a decrease in pain intensity in both groups. Adding tensioning NM to a traditional physical therapy program did not significantly decrease mechanosensitivity or pain intensity in comparison with the control group, who received only a traditional program.

Despite the lack of a significant difference in reducing mechanosensitivity of brachial nerve roots between the 2 groups, the analysis of data suggests greater clinical benefits for the study group.

In a systematic review with a meta-analysis, the effect of NM on nerve-related chronic musculoskeletal pain conditions was reviewed and it was concluded that NM was not superior to other interventions in decreasing pain or disability but it might be superior to minimal intervention in these regards [18]. These results are in accordance with the present study with reference to the benefit of adding NM to a multimodal program.

Also, the results of the current study agree with those obtained by Langevin et al. [19], who reported that only manual therapy and exercises were effective in reducing pain and functional limitations caused by CR, and NM led to no significant additional benefits. This study is comparable with the present one in the multimodal approach adopted and in including manual therapy in both groups.

Furthermore, Marks et al. [20] reported a significant difference between the NM group (nerve tensioner depending on the most painful test) and the cervical spine mobilization group (control group) in patients with cervicobrachial pain, favouring the control group regarding the improvement of cervical range of motion and decrease in neck pain. This study is comparable with the present one in one of the outcome measures (ULTT-1) and in using the neural tensioning technique; although it indicated the superiority of cervical mobilization on NM, it supports the effectiveness of NM in decreasing pain and mechanosensitivity. This superiority may have been caused by the way of NM application, as it continued for 15 minutes on one session while in the present study it was intermittent and lasted over 3 weeks.

In contrast to the current results, some papers [11,21] demonstrated that the addition of NM to a multimodal program was more effective in CR management; a study con- ducted by Anwar et al. [11] concluded that the addition of neurodynamics to moist heat, mobilization, and isometric exercises resulted in a significant improvement in the disability. Although both the NM technique and treatment duration were not identified, this study may indicate the benefit of adding NM to a multimodal program.

Gupta and Sharma [21] compared NM in the form of median slider applied 3 sets of 10 repetitions with isometric exercise, posture, and advice to move regularly, and concluded that there was a better improvement in the NM group regarding neck disability index, visual analogue scale, and painfree elbow extension. However, these results are not conclusive as the research compared NM with minimal intervention (only isometric exercise and advice), which may have contributed to the outcome.

The current study showed no significant effect of tensioning NM in spite of its proven impact on decreasing pain and sensory descriptors (stinging, tingling, tightness, sharpness, and numbness) induced by neurodynamic testing in the asymptomatic population [17]. This might be due to the relatively short duration, which may have limited our capacity to demonstrate a significant between-group difference, as well as to the absence of follow-up, which might have shown a significant difference in the long term.

Also, it might be more suitable to apply another NM technique in symptomatic patients (i.e. sliding NM) and to conduct separate NM on each peripheral nerve instead of mobilizing the entire brachial plexus.

All diagnoses of CR were included in the study and it might be more appropriate to include patients who most likely would benefit from NM. Finally, the absence of a real control group or no-treatment group might have affected our capacity to show a significant difference of NM.

\section{Limitations}

The results might have been affected by different causes of $\mathrm{CR}$ with variable symptom durations, as well as by life stresses, emotional status, and culture, which are known to influence pain coping strategy.

Further studies are recommended on specific groups of radiculopathy, with similar or at least comparable symptom durations. Also, a third group of no treatment as a real control group would be advised, and a long-term follow-up. Moreover, further studies could be conducted on other types of NM exercises or in comparison with mechanical traction.

\section{Conclusions}

In accordance with the current results, the addition of tensioning NM to traditional physical therapy yielded no signifi- 
cant additional benefits, although both groups showed reduced pain intensity and decreased mechanosensitivity after treatment.

\section{Clinical implications}

Traditional physical therapy alone is effective in the management of chronic unilateral CR. NM may be more beneficial for patients with increased mechanosensitivity of brachial nerves.

\section{Acknowledgments}

We would like to thank all individuals who contributed to the completion of this work, especially the study participants.

\section{Disclosure statement}

No author has any financial interest or received any financial benefit from this research.

\section{Conflict of interest}

The authors state no conflict of interest.

\section{References}

1. Caridi JM, Pumberger M, Hughes AP. Cervical radiculopathy: a review. HSS J. 2011;7(3):265-272; doi: 10.1007/ s11420-011-9218-z.

2. Butler DS. Adverse mechanical tension in the nervous system: a model for assessment and treatment. Aust $\mathrm{J}$ Physiother. 1989;35(4):227-238; doi: 10.1016/S00049514(14)60511-0.

3. Leoni D, Storer D, Gatti R, Egloff M, Barbero M. Upper limb neurodynamic test 1 on healthy individuals: intraand intersession reliability of the angle between pain onset and submaximal pain. Pain Res Manag. 2016;2016: 9607262; doi: 10.1155/2016/9607262.

4. Sharma S, Balthillaya G, Rao R, Mani R. Short term effectiveness of neural sliders and neural tensioners as an adjunct to static stretching of hamstrings on knee extension angle in healthy individuals: a randomized controlled trial. Phys Ther Sport. 2016;17:30-37; doi: 10.1016/j. ptsp.2015.03.003.

5. Shacklock M. Clinical neurodynamics: a new system of musculoskeletal treatment. Edinburgh: Elsevier Health Sciences; 2005.

6. Coppieters MW, Butler DS. Do 'sliders' slide and 'tensioners' tension? An analysis of neurodynamic techniques and considerations regarding their application. Man Ther. 2008;13(3):213-221; doi: 10.1016/j.math.2006.12.008.

7. Coppieters MW, Alshami AM. Longitudinal excursion and strain in the median nerve during novel nerve gliding exercises for carpal tunnel syndrome. J Orthop Res. 2007; 25(7):972-980; doi: 10.1002/jor.20310.

8. Ellis RF, Hing WA. Neural mobilization: a systematic review of randomized controlled trials with an analysis of therapeutic efficacy. J Man Manip Ther. 2008;16(1):8-22; doi: 10.1179/106698108790818594.

9. Wainner RS, Fritz JM, Irrgang JJ, Boninger ML, Delitto A, Allison S. Reliability and diagnostic accuracy of the clinical examination and patient self-report measures for cervical radiculopathy. Spine. 2003;28(1):52-62; doi: 10.1097/00007632-200301010-00014.

10. Sambyal S, Kumar S. Comparison between nerve mobilization and conventional physiotherapy in patients with cervical radiculopathy. Int J Innov Res Develop. 2013;2(8): $442-445$.
11. Anwar S, Malik AN, Amjad I. Effectiveness of neuromobilization in patients with cervical radiculopathy. Rawal Med J. 2015;40(1):34-36.

12. Schmid AB, Brunner F, Luomajoki H, Held U, Bachmann LM, Künzer S, et al. Reliability of clinical tests to evaluate nerve function and mechanosensitivity of the upper limb peripheral nervous system. BMC Musculoskelet Disord. 2009;10(1):11; doi: 10.1186/1471-247410-11.

13. Kannabiran B, Kumar S, Nagarani R. Neural mobilization for brachial neuralgia among cellulo-teno periosteo myalgic syndrome (CTPMS) patients. MOJ Orthop Rheumatol. 2015;2(1):00033; doi: 10.15406/mojor.2015.02. 00033.

14. Chiu TTW, Lam T-H, Hedley AJ. A randomized controlled trial on the efficacy of exercise for patients with chronic neck pain. Spine. 2005;30(1):E1-E7; doi: 10.1097/01. brs.0000149082.68262.b1.

15. Goyal M, Kumar A, Sethi R. Functional disability and grip strength of cervical radiculopathy patients before and after cervical collar use and traditional physiotherapy treatment. J Exerc Sci Physiother. 2012;8(2):119-122.

16. Butler DS. Mobilisation of the nervous system. Elsevier Health Sciences; 1991.

17. Beneciuk JM, Bishop MD, George SZ. Effects of upper extremity neural mobilization on thermal pain sensitivity: a sham-controlled study in asymptomatic participants. J Orthop Sports Phys Ther. 2009;39(6):428-438; doi: 10.2519/jospt.2009.2954.

18. Su Y, Lim ECW. Does evidence support the use of neural tissue management to reduce pain and disability in nerverelated chronic musculoskeletal pain? A systematic review with meta-analysis. Clin J Pain. 2016;32(11):9911004; doi: 10.1097/ajp.0000000000000340.

19. Langevin P, Desmeules F, Lamothe M, Robitaille S, Roy JS. Comparison of 2 manual therapy and exercise protocols for cervical radiculopathy: a randomized clinical trial evaluating short-term effects. J Orthop Sports Phys Ther. 2015;45(1):4-17; doi: 10.2519/jospt.2015.5211.

20. Marks M, Schöttker-Königer T, Probst A. Efficacy of cervical spine mobilization versus peripheral nerve slider techniques in cervicobrachial pain syndrome - a randomized clinical trial. J Phys Ther. 2011;4(1):9-17.

21. Gupta R, Sharma S. Effectiveness of median nerve slider's neurodynamics for managing pain and disability in cervicobrachial pain syndrome. Indian J Physiother Occup Ther. 2012;6(1):127-132. 